\begin{tabular}{|c|c|c|c|c|c|c|}
\hline Versuchsdatum & \multicolumn{2}{|c|}{20.9 .45}$. & \multicolumn{2}{|c|}{13.10 .45 . } & \multicolumn{2}{|c|}{22.10 .45 . } \\
\hline Pollenpflanzen ...... & $166 \mathrm{~K}$ & $166 \mathrm{~K}$ & $166 \mathrm{~K}$ & $166 \mathrm{~K}$ & $166 \mathrm{~K}$ & $166 \mathrm{~K}$ \\
\hline Griffelpflanzen ..... & $166 \mathrm{~K}$ & $166 \mathrm{H}$ & $166 \mathrm{~K}$ & $166 \mathrm{H}$ & $166 \mathrm{~K}$ & $166 \mathrm{H}$ \\
\hline Leitgewebezusatz von ... & $\begin{array}{c}166 \mathrm{~K} \\
=\text { eign. }\end{array}$ & $\begin{array}{c}166 \mathrm{H} \\
=\text { fremd. }\end{array}$ & $\begin{array}{c}166 \mathrm{~K} \\
=\text { eign. }\end{array}$ & $\begin{array}{c}166 \mathrm{H} \\
=\text { fremd. }\end{array}$ & $\begin{array}{c}166 \mathrm{~K} \\
=\text { eign. }\end{array}$ & $\begin{aligned} & 166 \mathrm{H} \\
= & \text { fremd. }\end{aligned}$ \\
\hline $\begin{array}{c}\mathbf{M} \text { des größten Wachstums } \\
\pm 3 \mathrm{~m} \text { in } \mathrm{mm}\end{array}$ & $\begin{array}{l}2,5 \\
\pm 0,5\end{array}$ & $\begin{array}{l}4,4 \\
\pm 1,2\end{array}$ & $\begin{array}{l}1,53 \\
\pm 0,36\end{array}$ & $\begin{array}{c}3,87 \\
\pm 1,86\end{array}$ & $\begin{array}{c}1,83 \\
\pm 0,37\end{array}$ & $\begin{array}{l}3,83 \\
\pm 2,20\end{array}$ \\
\hline
\end{tabular}

Tab. 1. Ergebnisse von drei Pollenschlauch-Wachstumsversuchen unter Verwendung von Wh i t e - Lösung. $166 \mathrm{~K}$ und $166 \mathrm{H}$ sind die Kennzeichen der benützten kreuzungsfertilen Petunien.

bedingenden Prinzips aufgefunden werden kann. Auf die Versuche, die an anderen Objekten zur Entscheidung der Frage „Hemmungsmechanismus oder Förderungsmechanismus"durchgeführt wurden, soll in einer ausführlichen Mitteilung eingegangen werden.

\title{
Selektiv-Filterung des Elektrenkephalogramms
}

\author{
Von Johannes Prast und Bruno Franek ${ }^{1}$ \\ (Z. Naturforschg. 1, 291-297 [1946]; eingegangen am 18. März 1946)
}

\begin{abstract}
Gegenüber den bisherigen Versuchen einer nachträglichen Frequenzanalyse des aufgenommenen EEG wird in dem beschriebenen Verfahren durch selektive, elektrische Filterung jede Einzelfrequenz für sich isoliert und dann aufgezeichnet. Auf diese Weise konnten mehrere, verschiedene Frequenzen ihren quantitativen wie qualitativen Eigenschaften nach unterschieden, und frequenzisolierte, zeitlich und örtlich in Zusammenhang mit dem Reiz stehende Hirnaktionsschwingungen betrachtet werden. Mit der Erschließung höherfrequenter Bereiche für die analytische Forschung werden die Feinheiten des EEG erst der Möglichkeit einer Deutung zugeführt.
\end{abstract}

$\mathrm{D}_{\mathrm{P}}^{\mathrm{ie}}$ ie von der Kopfhaut abgeleiteten elektrischen Potentialschwankungen sind heute soweit bekannt, daß sie nicht nur wertvolle Hinweise bei der Erforschung derVorgänge im Zentralnervensystem bieten, sondern auch schon für die klinische Untersuchung von Hirnschädigungen herangezogen werden $^{2}$. In beiden Anwendungen sind noch wesentliche Fortschritte durch weitere gründliche Erforschung der Feinheiten und Einzelheiten des Elektrenkephalogramms (EEG) und durch Einsatz leistungsfähiger Meßverfahren zu erwarten.

Seit den ersten graphischen Registrierungen der Potentialschwankungen des Gehirns bestanden zwei Hauptschwierigkeiten; 1. war die Aufgabe einer möglichst wirklichkeitsgetreuen graphischen Wiedergabe zu lösen, was dank ausgezeichneter technisch-physikalischer Methoden im Laufe der

1 Die Untersuchungen wurden von B. F r a n e k 1942/43 in der Physiologischen Abteilung des Kaiser-WilhelmInstituts für Hirnforschung und 1944/45 in der Neurophysiologischen Abteilung der Universitäts-Nervenklinik Wien nach einer 1942 von J. P r a s t entwickelten Methodik durchgeführt.
Zeit weitgehend gelang; 2. die Frage einer geeigneten Interpretation des erhaltenen Kurvenbildes ${ }^{3}$.

Ein unter den üblichen Bedingungen aufgenommenes EEG zeigt einen recht unübersichtlichen Kurvenverlauf. Bei der Auswertung wurde im allgemeinen nur auf den zeitlichen Ablauf der deutlichsten Schwankungen und deren krasseste. Veränderungen geachtet. Die Frequenz wurde durch Auszählung der Nulldurchgänge oder der positiven bzw. negativen Spitzen ermittelt. Dabei zeigte sich, daß die größten und am häufigsten auftretenden Schwankungen etwa einer Frequenz von $10 \mathrm{~Hz}$ entsprechen $\left(\alpha\right.$-Wellen, B erge $\left.\mathbf{r}^{4}\right)$. In Zeiten, die weitgehend von $\alpha$-Wellen frei sind, kann man außerdem Schwankungen einer Frequenz von $20 \mathrm{~Hz}$ auszählen. ( $\beta$-Wellen. Wir bezeichnen mit Kornmüller nur die $20-\mathrm{Hz}-$ Schwingungen als $\beta$-Wellen, alle höherfrequenten Schwingungen werden direkt durch ihre Frequenz gekennzeichnet.)

Neben den $\alpha$ - und $\beta$-Wellen enthält das EEG noch viele Schwingungen höherer Frequenzen. Diese sind den

2 Die Literatur hierzu bei: A. E. K ornmüll er, Klinische Elektrenkephalographie, München-Berlin J. F. Lehmann 1944.

${ }^{3}$ Hierzu s. z. B.: A. E. K or n müller u. R. J a n z en, Arch. Psychiatrie 110, 237 [1939].

* Nova acta Leopoldina, N. F. 6, 191 [1938] ; 6, 296. 
$\boldsymbol{\alpha}$ - und $\beta$-Wellen überlagert, von kleiner Amplitude und, ebenso wie die $\beta$-Wellen in Zeiten starker $\alpha$-Wellenproduktion, meist nicht ohne weiteres auszuzählen und zu erkennen.

Das EEG ist eine zusammengesetzte Kurve, die Resultierende verschiedener Einzelschwingungen. Es ist bekannt, daß eine Anzahl völlig regelmäßig verlaufender Einzelvorgänge in der Superposition ein verwirrendes, unregelmäßiges Bild geben können, in dem man die einzelnen Komponenten meist nicht mehr erkennen kann. Aus diesem Grund wird beispielsweise in der Akustik ein Vokal nicht etwa durch den komplizierten Ablauf des entstehenden Schalldruckes, sondern durch die Einzelschwingungen, aus denen er sich aufbaut, beschrieben ${ }^{5}$.

Die bisherige Erforschung des EEG war im wesentlichen eine Erforschung der hervortretenden $\alpha$-Welle, während über die überlagerten höherfrequenten Komponenten und ihre biologische Bedeutung bisher wenig ausgesagt wurde. Wenn wir nicht der Gefahr verfallen wollen, nur einen kleinen Teil dessen zu betrachten, was uns das EEG darbietet, so müssen wir uns mehr dessen bewußt werden, daß wir eine zusammengesetzte Schwingung aufnehmen, deren Einzelkomponenten für sich nicht ohne weiteres erfaßt werden. Wir müssen nachträglich aus diesem Frequenzgemisch die Einzelschwingungen isolieren. Möglich ist eine solche Isolierung für alle Einzelschwingungen, die mit verschiedenen Frequenzen auftreten. Den Hauptnutzen eines solchen Vorgehens erwarten wir nicht von der dadurch möglichen Aufstellung eines Frequenz-Spektrums, sondern von der isolierten Darstellung und Betrachtung der Einzelschwingungen, von der Feststellung ihres unterschiedlichen Verhaltens gegenüber der $\alpha$-Welle und untereinander, von den Zusammenhängen der Einzelschwingungen mit Sinnesvorgängen und äußeren Beeinflussungen. Durch gleichzeitige Erfassung mehrerer verschiedener Einzelschwingungen können Komponenten mit spezifischem Verhalten leicht von solchen unterschieden werden, die mathematisch und biologisch nur eine „Oberwelle“, nur eine verzerrende Begleiterscheinung der Grundwelle darstellen.

\section{Methoden}

Methodisch stehen zwei Wege zur Erfassung der Einzelschwingungen zur Verfügung: Entweder

- Klangspektren von Vokalen bei: F. Trendelenburg, Klänge und Geräusche, Berlin Springer 1935, S. 82.

B Pflügers Arch. ges. Physiol. Menschen, Tiere 230, 106 [1932]. wird ein normales EEG in üblicher Weise aufgezeichnet, das nachträglich rechnerisch oder mit Hilfe geeigneter Apparate in seine Einzelschwingungen zerlegt wird, oder die Einzelschwingungen werden zuvor durch geeignete Filter isoliert und dann jede für sich aufgezeichnet.

$\mathrm{Zu}$ der ersten Gruppe gehört z. B. der Versuch einer rechnerischen Fourier-Analyse einer EEG-Schwingung durch G. Dietsch 19326. Dietsch setzte als Grundkomponente $10 \mathrm{~Hz}$ (bzw. 7 und $5 \mathrm{~Hz}$ in pathologischen Fällen) und konnte deshalb Schwingungen mit einer, ein geradzahliges Vielfaches der angenommenen Grundkomponente bildenden Frequenz berechnen. Unter dieser Annahme konnte er aber nicht das tatsächliche Spektrum des EEG bilden, das auch Komponenten enthält, die unharmonisch zu der angenommenen Grundwelle von $10 \mathrm{~Hz}$ liegen. $\mathrm{H}$. R o h r a cher berichtet über weitere Fourier-Analysen des EEG ${ }^{7}$, wobei aber die Komponenten höherer Frequenz durch Umzeichnen von vornherein ausgeschaltet wurden, so daß eine Diskussion der Ergebnisse nicht im Rahmen dieser Arbeit liegt. L. J. Franke und L. J. K o o pman $\mathrm{n}^{8}$ haben ebenfalls Fourier-Analysen des EEG in ähnlicher Weise wie Dietsch durchgeführt. Über Fourier-Analysen der EEG von Tieren berichtet M. N. Livanow ${ }^{\boldsymbol{\theta}}$. - Zur Durchführung einer einwandfreien Fourier-Analyse des EEG darf die Grundkomponente mit maximal $1 \mathrm{~Hz}$ angesetzt werden. Zur Erfassung des Bereiches bis $100 \mathrm{~Hz}$ wären also 100 Oberwellen zu berechnen. Eine rechnerische Fourier-Analyse einer einzelnen Schwingungsperiode bis zur 10. Oberwelle beansprucht einen geübten Mathematiker etwa einen halben Tag ${ }^{\mathbf{1 0}}$, eine Analyse bis zur 100. Oberwelle ist praktisch kaum durchführbar. Mit dem nötigen immensen Arbeitsaufwand würde ja auch weiter nichts erreicht werden als eine mathematisch exakt in Zahlen ausgedrückte Aussage über die Form einer einzigen Schwingungsperiode, die als typisch hingestellt wird. Erkenntnisfördernde Aufschlüsse versprechen wir uns nicht davon, sondern von dem Studium isolierter Einzelschwingungen in all ihren Veränderungen.

$\mathrm{Zu}$ den Verfahren der ersten Gruppe gehört ferner die Arbeitsmethode von A. M. Grass und F. A. Gibbs, die ihr EEG zu einer Schattenkurve auf einem Film präparieren. Diesen Film lassen sie zwischen einer Lichtquelle und einer Photozelle durchlaufen, so daß eine Modulation des Lichtstromes im Sinne des EEG entsteht. Die an der Photozelle entstehenden Potentialschwankungen werden auf ein Filter gegeben, das jeweils nur eine

7 Pflügers Arch. ges. Physiol. Menschen, Tiere 238, 535 [1937]; 240, 191 [1938].

8 Z. Neurol. Psychiatrie 162, 278 [1938].

9 Ref. in Zbl. ges. Neurol. u. Psychiatrie 92, 118 [1939].

10 F. Trendelenburg u. E. Franz, Z. techn. Physik 16, 513 [1935]. Über Durchführung von F o u r i e r Analysen z. B.: G. Joos u. Th. Kaluza, Höhere Mathematik für Praktiker, Leipzig Johann Ambrosius Barth 1942, oder Handb. d. Physik, Bd. VIII. Berlin Springer, oder bei G. Dietsch, Pflügers Arch. ges. Physiol. Menschen, Tiere 230, 106 [1932]. 
Frequenz überträgt ${ }^{11}$. Ähnliche Apparaturen wurden 1924 von C.F.Sicia ${ }^{12}$ und 1932 von Dietsch und Frick $\mathrm{e}^{\mathbf{1 3}}$ angegeben. Voraussetzung für einwandfreie Ergebnisse bei dieser Methodik ist, daf bei der Umwandlung in Lichtmodulation und Rückwandlung in Spannungsschwankungen an der Photozelle keine Verzerrungen auftreten. Verzerrungen, die vor dem Filter entstehen, bedeuten Hinzutreten neuer Oberwellen und damit Falschmessung. Diese Gefahr ist übrigens mit allen Verfahren verbunden, bei denen zuerst ein EEG aufgezeichnet und danach zerlegt wird. Gewisse kleine Verzerrungen bei der Aufzeichnung lassen sich nicht vermeiden. Diese Fehler kommen aber leicht in die Größenordnung der im EEG enthaltenen höherfrequenten Komponenten. Fehler dieser Größenordnung sind schon wegen der endlichen Strichstärke der aufgezeichneten Kurve nicht zu vermeiden. Uber Frequenz-Analysen des EEG mittels des von A. E. D o u g las angegebenen Cycloskops berichtet $\mathrm{R}$. Coh $\mathrm{n}^{\mathbf{1 4}}$.

Bei Verfahren der zweiten Art, bei denen das EEG vor der Aufzeichnung in seine Einzelschwingungen zerlegt wird, können derartige Fehler bei der Auswertung schwacher Komponenten nicht entstehen. Alle Verzerrungen, die nach dem Auseinanderfiltern der Einzelschwingungen entstehen, können an der einmal festgestellten Verteilung der Komponenten im Spektrum nichts mehr ändern. Aus diesem Grunde wird man das Filter auch möglichst weit an den Verstärkereingang legen. Auch besonders schwache Komponenten können dann nach Durchlaufen des Filters entsprechend verstärkt und sichtbar gemacht werden. Die ersten Aufnahmen nach einem derartigen Verfahren dürfte Z. Drohocki 1938 durchgeführt haben ${ }^{15}$.

Weitere hierher gehörige EEG-Aufnahmen unter Verwendung elektrischer oder mechanischer Resonanzsysteme bei der Verstärkung oder Aufzeichnung wurden von Davis, Davis, Loomis, Harvey, Hobart ${ }^{16}$ und von $\mathrm{Parr}$ und Walte $\mathrm{r}^{17}$ mitgeteilt. In einem von W. G. Walter beschriebenen Gerät werden nicht die einzelnen Schwingungen als Kurve aufgezeichnet, sondern die Amplituden der Einzelschwingungen werden nacheinander in einem $10 \mathrm{sec}$ dauernden Zyklus gemessen und als Striche entsprechender Länge auf vorbeilaufendem Papier markiert ${ }^{18}$. Das Gerät wurde für den Bereich von 2 bis $22 \mathrm{~Hz}$ entwickelt. Die Brauchbarkeit dieses Verfahrens wird durch die Langsamkeit des Meß- und Aufzeichnungs-

11 J. Neurophysiol. 1, 521 [1938].

12 Journ. opt. Soc. America 9, 487 [1924].

13 Elektr. Nachr. Techn, 9, 9 [1932].

14 Ref. in Zbl. ges. Neurol. Psychiatrie 104, 531 [1943].

15 C. R. Séances Soc. Biol. Filiales Associées 129, 889 [1938]; Klin. Wschr. 18, 536 [1939]. zyklus in Frage gestellt. Viele Komponenten, wie z.B. die 22-Hz-Schwingung, zeigen schon in einem Zeitraum von 0,2 sec starke Amplitudenveränderungen; innerhalb einer Zeit von 10 sec kann wohl kaum jemals mit konstant bleibenden Verhältnissen gerechnet werden. Walter analysiert also die 22-Hz-Komponente einer Schwingung, diegar nicht mehr die gleiche ist wie die, von der die $2-\mathrm{Hz}-\mathrm{Kom}$ ponente ermittelt wurde.

Die Ausnutzung der Resonanz mechanischer Biegeschwingungen einseitig eingespannter Blattfedern hat gegenüber normalen elektrischen Schwingungskreisen den Nachteil der kleineren Frequenzeindeutigkeit. Solche Resonanzsysteme haben, ebenso wie die in der Ultrakurzwellentechnik verwendeten abgestimmten Leitungen, nicht nur eine, sondern mehrere Resonanzfrequenzen, die sich zur tiefsten, erregbaren Resonanzfrequenz $3: 1,5: 1,7: 1,9: 1,11: 1$ usw. verhalten. Durch möglichst punktförmige Konzentrierung der Masse am freien, schwingenden Federende treten die höheren Resonanzfrequenzen zurück; praktisch bleibt die Frequenzeindeutigkeit aber immer unter der eines elektrischen Schwingungskreises mit konzentrierten Kapazitäten und Induktivitäten. Die „Bandbreite“ einer schwingenden Feder ist von Natur aus allerdings kleiner, muß aber aus Gründen der Anschwingzeit künstlich durch Dämpfung auf größere Werte gebracht werden. Da auch die Betriebssicherheit von Geräten, die mechanische Bewegungen vermeiden, stets größer ist, dürfte die Aussiebung mittels elektrischer Filter das sauberste Vorgehen darstellen.

Wir konnten eine Reihe regelmäßig wiederkehrender Eigentümlichkeiten der höherfrequenten Komponenten feststellen und eine gewisse wohlgeordnete Gliederung dieses Frequenzbereichs ermitteln. Das bei diesen Untersuchungen verwendete einfache und genau arbeitende Verfahren zur Isolierung der Einzelschwingungen, das eine Bearbeitung umfangreichen Materials ermöglicht, und mit dem neben quantitativen Vergleichen auch qualitative Eigentümlichkeiten erkannt werden können, ist folgendes:

16 J. Neurophysiol. 1, 24 [1938], Aussiebung der 3,10 und $14 \mathrm{~Hz}-\mathrm{Komponente}$ bei Schlaf.

17 Electronic Engineering XV, 456 [1943]; XV, 519 [1943].

18 Electronic Engineering XVI, 9 [1943]. Für einen speziellen Zweck hat Kornmüller mit Fa. DTW. ein Gerät zur laufenden Signalisierung einiger langsamer Schwingungen durch mechanische Resonanzsysteme entwiickelt (unveröffentlicht). 


\section{Selektivfilter-Verfahren}

Durch ein zwischengeschaltetes abstimmbares Filter wird der normale Utbertragungsbereich des zur Aufnahme benutzten Gerätes so eingegrenzt, daß von den vielen Einzelschwingungen, aus denen sich das EEG zusammensetzt, jeweils nur eine, der Einstellung des Filters entsprechende Schwingung durchgelassen wird. Da die Einstellung des Filters sich über das ganze Spektrum verändern läßt, kann ohne weiteres jede Einzelschwingung nachgewiesen und untersucht werden. Das Filter besteht aus einem frequenzunabhängigen Längswiderstand und einem Querwiderstand, der bei der Resonanzfrequenz ein Maximum des Widerstandswertes be-

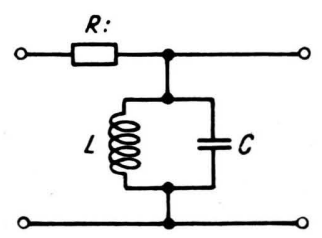

Abb. 1. Ersatzschaltbild des Filters.

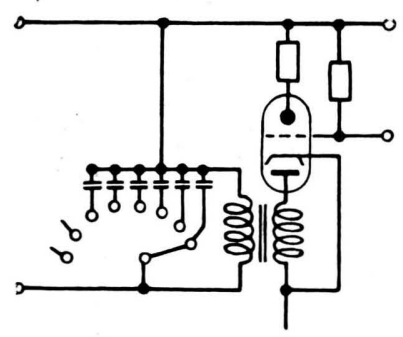

Abb. 2. Schaltung. sitzt. Der Längswiderstand $R i$ ist durch den Innenwiderstand einer Elektronenröhre gebildet, der Querwiderstand ist ein Schwingkreis, welcher aus der Induktivität $L$ und der parallel geschalteten Kapazität $C$ gebildet ist (Abb. 1 u. 2). Bei der Resonanzfrequenz $\frac{1}{2 \pi \sqrt{L \cdot C}}$ hat der Schwingkreiswiderstand den maximalen Wert $\Re=\frac{L}{r \cdot C}$ ( $r=$ Ohmscher Widerstand der Schwingkreisspule). Unter der Bedingung, daß $R i$ sehr groß gegen $\Re$ ist, was bei Verwendung einer Röhre mit Schirmgitter gegeben ist, hat die Ausgangsspannung als Funktion der Frequenz denselben Verlauf wie der Schwingkreiswiderstand. Das Filter überträgt die Resonanzfrequenz gut und unterdrückt die Nachbarfrequenzen entsprechend.

Die Bandbreite des Filters ist veränderlich. An sich ist aus Selektivitätsgründen eine extrem kleine Bandbreite erwünscht. Andererseits setzt die Natur hier Grenzen durch die Einschwingzeit. Eine plötzliche Veränderung der Schwingung wird nämlich nur dann naturgetreu übertragen, wenn die Bandbreite unendlich groß ist. Bei endlicher Bandbreite schwingt das Ǔbertragungssystem, also das Filter, erst allmählich von dem ursprünglichen

19 K. Küpfmüller, Elektr. Nachr.-Techn. 5, 18 [1928]. in den geänderten Zustand ein. Die dazu benötigte Zeit, die sogenannte Einschwingzeit, beträgt $T=1 / \Delta f^{19}(\Delta f=$ Halbwertsbreite). Die kleinste einstellbare Bandbreite ist bestimmt durch das Verhältnis des $\mathrm{O} h \mathrm{~m}$ schen Widerstandes der Schwingkreisspule zur Induktivität derselben:

$\frac{r}{2 \pi \cdot L}$. Es kommt also darauf an, das Verhältnis $L / r$, die sogenannte Zeitkonstante der Spule, groß zu machen, was durch Verwendung großer Querschnitte für den Eisenkern zu erreichen ist. In einer gegenüber Abb. 2 etwas abgeänderten Schaltung können Eisenbleche mit großer Anfangspermeabilität (Permalloy, Megaperm, Permenorm u. a.) verwendet werden. In diesem Fall ist die kleinste gebräuchliche Kerngröße M $42(42 \times 42 \times$ $18 \mathrm{~mm}$ ) ausreichend. Bei höheren Frequenzen wird die Bandbreite noch durch den dämpfenden Einfluß der Eisenverluste vergrößert. Diese lassen sich durch Verwendung dünner Bleche klein halten. Eine Vergrößerung der Bandbreite wird erreicht durch Einschaltung zusätzlicher Widerstände in Reihe zur Schwingkreisspule. Bandbreiten unter etwa $1 \mathrm{~Hz}$ sind für die hier diskutierten Zwecke unbrauchbar, da Anschwingzeiten von mehr als 1 sec im allgemeinen das Kurvenbild zu stark verfälschen. Wir haben allgemein mit einer Bandbreite von etwa $2 \mathrm{~Hz}$ gearbeitet. Für die Form des Übertragungsbandes ist erwünscht, daß ein schmales Band gleichmäßig gut übertragen wird und beidseitig davon überhaupt nichts, $d$. h. die Übertragungskurve soll rechteckförmig verlaufen. Diese Bedingung ist bei den ersten ausgeführten Filtern nur grob angenähert erfüllt. Es ging bei diesen Geräten darum, eine Bedienung durch elektrotechnisch nicht vorgebildete Kräfte und größtmögliche Betriebssicherheit zu erreichen. Mit entsprechend größerem Aufwand lassen sich ohne weiteres Filter erstellen, die mehr der Rechteckbedingung nahe kommen ${ }^{20}$. Es wurde jedoch davon Abstand genommen, da größerer Aufwand im allgemeinen Vergrößerung der Fehlerquellen bedeutet und der Einschwingvorgang eines rechteckförmigen Übertragungssystemes komplizierter verläuft ${ }^{21}$. Die „Verstärkung" des Filters ist für die einzelnen Frequenz-Einstellungen proportional dem Quadrat der Frequenz. Das ergibt sich, wenn die Schwingkreiskapazität umgeschaltet wird und die Induktivität konstant bleibt. In diesem Fall ist bei Schwingkreisen bis etwa $50 \mathrm{~Hz}$ auch für alle Frequenz-Einstellungen die Bandbreite konstant. Bei der Einstellung auf $10 \mathrm{~Hz}$ ist die Verstärkung zu etwa 1 gewählt worden ${ }^{22}$.

Beim normalen EEG macht sich bei der Aufnahme sehr schwacher EEG oft schon das Eigenrauschen störend bemerkbar. Es könnte angenommen werden, daß bei Aufnahme höherfrequenter Komponenten, die mit einer Amplitude von nur wenigen Prozent der Grundschwin-

20 Hierüber s. z. B.: R. Feldtkeller, Einführung in die Theorie der Rundfunk-Siebschaltungen $u$. R. Feld t keller, Einführung in die Siebschaltungstheorie. Leipzig H ir z el 1940, 1942.

21 F. Str e c k e r, Elektr. Nachr.-Techn. 17. 17 [1934], u. O. E r h a r d t. Elektr. Nachr.-Techn. 11, 267 [1934].

${ }_{22}$ Die Amplitude der Einzelkomponenten fällt bis etwa $50 \mathrm{~Hz}$ stark, eine mit der Frequenz größer werdende Verstärkung erspart in diesem Frequenzbereich Nachregeln der Aufnahmeverstärkung bei Umschalten der Filterfrequenz. 
gung auftreten, das Rauschen entsprechend mehr stört. Eine Unterdrückung solcher Einzelschwingungen sehr kleiner Intensität durch die Rauschspannung der Anfangsstufe ist jedoch nicht $\mathrm{zu}$ fürchten, da die Rauschamplitude neben der Dimensionierung der Anfangsstufe durch die Bandbreite bestimmt wird. Die Größe des Funkeleffekts, der in dem interessierenden Frequenzgebiet praktisch das gesamte Rauschen bestimmt, verläuft etwa proportional dem Reziprokwert der Frequenz.
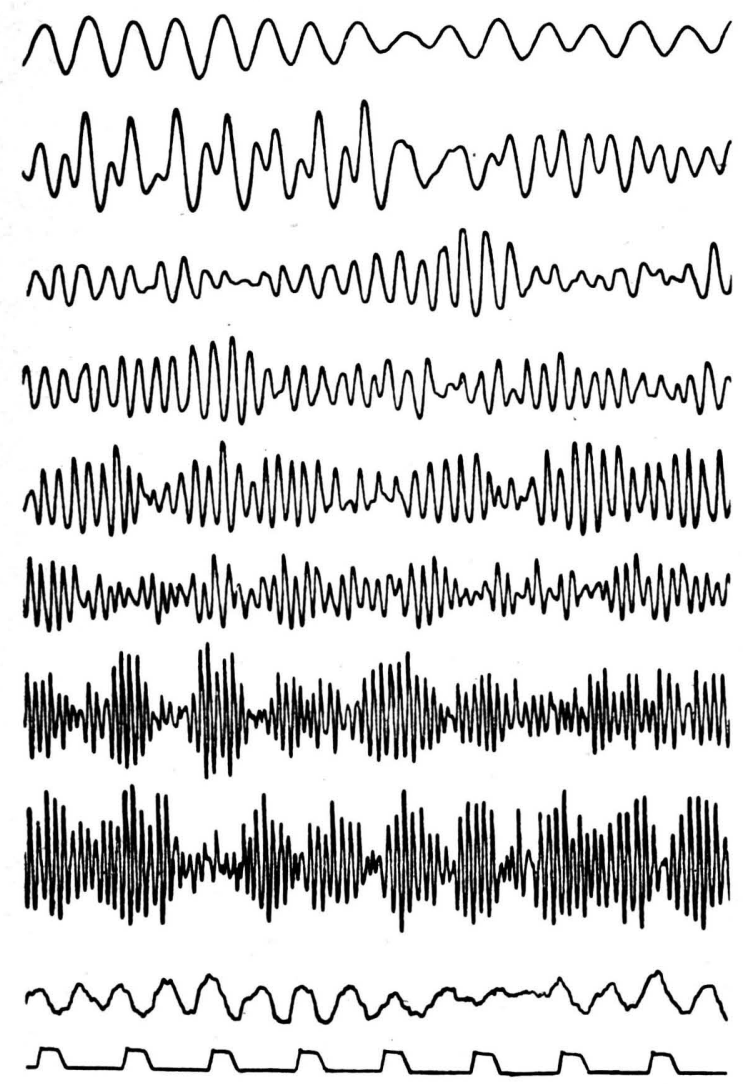

Abb. 3. Übersicht über einen Teil der selektiv gefilterten Frequenzen, wie sie im normalen EEG (unterste Kurve) enthalten sind. Zeitmarke: $1 / 5$ Sek.

Bei Übertragung eines schmalen Frequenzbandes verkleinert sich die Rauschamplitude, da die Anteile entfallen, die von den unterdrückten Frequenzen geliefert werden. Die Rauschamplitude wird um so kleiner, je mehr das Übertragungsband nach höheren Frequenzen verlegt wird, wo ja die Amplituden der Einzelschwingungen des EEG schwach werden ${ }^{23}$.

\section{Anwendung}

Der Wert eines Selektivfilter-Verfahrens für die analytische Forschung dürfte außer Frage stehen,

${ }^{23}$ Über Störungen durch das Eigenrauschen: H. R o th e u. W. K l e en, Elektronenröhren als Anfangsstufen-Verstärker. Leipzig Be cker \& Er ler 1944. soweit es die quantitative Bestimmung der Einzelkomponenten angeht. Wir konnten derart folgende Hauptschwingungen isoliert zur Darstellung bringen $^{24}$ (vergl. Abb. 3 u. 4) :

\begin{tabular}{r|r|r|r}
$10 \mathrm{~Hz}$ & $27 \mathrm{~Hz}$ & $47 \mathrm{~Hz}$ & $88 \mathrm{~Hz}$ \\
$18 \mathrm{~Hz}$ & $30 \mathrm{~Hz}$ & $52 \mathrm{~Hz}$ & $92-98 \mathrm{~Hz}$ \\
$20 \mathrm{~Hz}$ & $33-36 \mathrm{~Hz}$ & $57 \mathrm{~Hz}$ & $107 \mathrm{~Hz}$ \\
$22 \mathrm{~Hz}$ & $40 \mathrm{~Hz}$ & $69-79 \mathrm{~Hz}$ & $120 \mathrm{~Hz}$ \\
$\sim 25 \mathrm{~Hz}$ & $42 \mathrm{~Hz}$ & $82 \mathrm{~Hz}$ &
\end{tabular}

Eine ebenfalls gefundene $50-\mathrm{Hz}-\mathrm{Sch}$ wingung wurde wegen der Gefahr der Verwechslung mit eingestreutem technischem Wechselstrom von vornherein aus der Diskussion herausgenommen. In dem von Drohocki untersuchten Frequenzbereich stimmen die von uns gefundenen Frequenzen etwa mit seinen Werten überein.

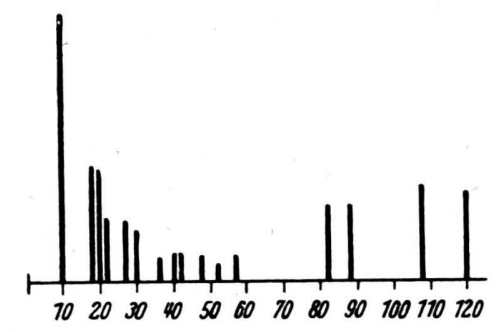

Abb. 4. Frequenzspektrum eines EEG.

Hinsichtlich der Art ihres Auftretens lassen sich die gefundenen Einzelschwingungen in drei Hauptgruppen einteilen:

$\begin{array}{ccc}\text { Gruppe A } & \text { Gruppe B } & \text { Gruppe C } \\ 10 \mathrm{~Hz} & 18 \mathrm{~Hz} & \text { um } 25 \mathrm{~Hz} \\ 20 \mathrm{~Hz} & 22 \mathrm{~Hz} & 33-36 \mathrm{~Hz} \\ 30 \mathrm{~Hz} & 27 \mathrm{~Hz} & 69-79 \mathrm{~Hz} \\ 40 \mathrm{~Hz} & 42 \mathrm{~Hz} & 92-98 \mathrm{~Hz} \\ & 47 \mathrm{~Hz} & \\ & 52 \mathrm{~Hz} & \\ & 57 \mathrm{~Hz} & \\ & 107 \mathrm{~Hz} & \end{array}$

Die Schwingungen der Gruppe $A$ zeichnen sich durch eindeutige, regionäre Unterschiede aus. Die bekannte Erscheinung, daß die 10-Hz-Schwingung occipital am stärksten auftritt und nach frontal zu an Amplitude und Regelmäßigkeit abnimmt ${ }^{25}$, konnten wir mit gefilterten $10-\mathrm{Hz}-\mathrm{Sch}$ wingungen eindeutig bestätigen.

Die 30-Hz-Schwingung verhält sich gleichartig. Die 20-Hz-Schwingung und in gleicher Weise die

24 B. Franek, Über eine Frequenzanalyse des Elektrenkephalogramms, Dissertat. Berlin 1944, u. B. F r a nek, Frequenzanalyse bioelektrischer Potentialschwankungen (diese Z. 1, 231 [1946]).

25 A. E. Kornmüller u. R. Janzen, Arch. Psychiatrie 166, 224 [1939]; R. J ung, Nervenarzt 12, 577 [1939]. 

sätzliches Verhalten, d. h. treten frontal am stärksten und regelmäßigsten auf, nach occipital zu immer schwächer und indifferenter ${ }^{26}$. Der $40-\mathrm{Hz}$ Komponente kommt sicher keine eigene biologische Bedeutung $\mathrm{zu}$, sie stellt als tatsächliche „Oberwelle" nur eine verzerrende Begleiterscheinung der 20-Hz-Schwingung dar, durch die ausgesagt ist. Das Gleiche gilt für die 30-Hz-Komponente bezüglich der 10-Hz-Schwingung.
40-Hz-Komponente zeigen ein polar dazu gegenwird, daß diese eben keine reine Sinusschwingung

Die Gruppe $C$ umfaßt Schwingungen, bei denen weder von einer Stabilität der Frequenz noch der Amplitude die Rede sein kann. Diese Schwingungen traten auch bei weitem nicht so sinusförmig wie die oben beschriebenen auf.

Abgesehen von der Gruppe C, die in ihrem ganz indifferenten Verhalten eine Sonderstellung einnimmt, sind beim Vergleich zahlreicher Frequenzspektren der gleichen Versuchsperson keinerlei Frequenzschwankungen festzustellen.-BeimVergleich zahlreicher Frequenzspektren verschiedener Versuchspersonen untereinander sind in etwa $70 \%$ der untersuchten Fälle ebenfalls keine . nennenswerten Frequenzschwankungen festzustellen. Bei den übrigen Vp. betrugen die Abweichungen der Frequenzen gegenüber dem Normaltyp bis $\mathrm{zu} \pm 2 \mathrm{~Hz}$.

Rückschlüsse hinsichtlich der Zuordnung der einzelnen Gruppen $\mathrm{zu}$ verschiedenen biologischen Vorgängen halten wir für verfrüht. Wahrscheinlich werden einige Schwingungen nicht das elektrische Bild von Hirnvorgängen

Die Gruppe $B$ umfaßt Schwingungen, bei denen keinerlei regionäre Unterschiede festgestellt werden konnten. Sie treten eruptiv, ohne jeden allmählichen Übergang beim Einsetzen und Ausklingen auf, erscheinen plötzlich in einer Schwingungsgruppe sehr konstanter Frequenz mit einer Amplitude, die die vorausgehende indifferente Kurve bei weitem überragt. Nach $0,2-0,5$ sec verschwindet diese eindeutige klare Schwingungsgruppe wieder schroff. Die Häufigkeit, mit der diese Schwingungsgruppen auftreten, nimmt mit höheren Frequenzen zu, ihre Länge und die Länge der indifferenten Zwischenstücke im gleichen Sinne ab. Die Frequenzstabilität und die Amplitudenkonstanz innerhalb der Schwingungsgruppe steigt mit höheren Frequenzen.

${ }^{26}$ Hierüber auch: A. L. Loomis, E. N. Harvey u. G. A. Hobar t, J. Neurophysiol. 1, 413 [1938]. darstellen. - In der Möglichkeit diskreter Erfassung und damit der Ausscheidung von „Störkomponenten" liegt gerade eine der Hauptstärken einer Aufnahme des EEG über Filter. Ein eindringliches Beispiel für die Leistungsfähigkeit des Verfahrens stellt die Isolierung und Beschreibung zweier so dicht beieinanderliegender Komponenten wie 20 und $22 \mathrm{~Hz}$ dar, die sich sowohl in der Form ihres Auftretens, wie auch bezüglich regionärer Unterschiede klar und eindeutig unterscheiden. Eine weitere wichtige Feststellung, die durch Selektivfilterung des EEG ermöglicht wurde, war die Auffindung eindeutiger Aktionsschwingungen auf Augenbelichtung beim Menschen (vergl. Abb. 5). Diese äußern sich in einem An- und Endeffekt, ähnlich wie sie K o r n m ü 1 le r bei Ableitung von freigelegten Tiergehirnen fand ${ }^{27}$. Bei Aufnahme 27 A. E. K or n müller, Die bioelektrischen Erschei- 
der $\alpha$-Komponente über Filter sieht man klare und eindeutige Schwingungsgruppen als An- und Endeffekt, die sich hinsichtlich Intensität und Dauer in so hohem Maße gleichen, daß man glaubt, im Endeffekt ein Spíegelbild des zugehörigen Aneffektes zu sehen. Diese klaren, frappierenden Bilder ergeben sich nur bei Aufnahmen mit Selektivfilterung. Im ungefilterten EEG ist nämlich der eindeutigen $\alpha$-Aktionsschwingung eine $20-\mathrm{Hz}-\mathrm{Ak}$ tionsschwingung überlagert, die wohl ebenfalls eine eindeutige Antwort auf das Ein- und Aussetzen der Augenbelichtung darstellt, aber nicht so-regelmäßig und charakteristisch wie die $\alpha$-Aktionsschwingung auftritt. Die Superposition der charakteristischen $\alpha$-Aktionsschwingung, der unregelmäßigen 20-Hz-Aktionsschwingung und der völlig unbeeinflußten Schwingungen der Gruppe B ergibt ein Kurvenbild, ähnlich den von Kornmüll er aufgenommenen Aktionsschwingungen bei Tieren, nur noch weniger ins Auge fallend. Die Aktionsschwingungen waren nur von einem eng umgrenzten Feld abzuleiten.

Bei der Betrachtung der fast ideal sinusförmigen Schwingungen, nicht nur der $\alpha$-Welle, sondern auch vieler anderer Komponenten; könnten dem, der an das ungefilterte EEG gewöhnt ist, Bedenken kommen, ob das Filter nicht die wirklichen Verhältnisse vergewaltigt, ob es nicht selbst Schwingungen erzeugt und zusetzt. Diese Befürchtungen werden sich verstärken wegen der großen Zahl der Einzelschwingungen, die das EEG enthält. Elektrisch so einfache Gebilde wie die verwendeten Filter sind übersichtlich genug, als daß sie irgendwelche unkontrollierbaren „wilden Schwingungen“ erzeugen könnten. Wohl wird es manchen stutzig machen, daß eine solche Filterschaltung auf rechteckige Spannungsstöße mit einwandfreien Sinusspannungen antwortet. Diese Sinusschwingungen sind aber Bestandteile des rechteckigen Spannungsstoßes, dessen Frequenzspektrum aus dicht beieinander liegenden Komponenten besteht. Aus diesem Spektrum wird mit dem Filter die Resonanzfrequenz desselben herausgesiebt ${ }^{28}$. Das „Anstoßen eines Schwingungskreises zu gedämpften Schwingungen" ist nur eine andere, etwas unglückliche Bezeichnung für den gleichen physikalischen Vorgang ${ }^{29}$.

Freilich kann nicht jede von der Kopfhaut über dem Gehirn aufgenommene und aus dieser Kurve herausgefilterte Einzelschwingung als ein Substrat der Hirntätigkeit angesehen werden. Es wäre z. B. naheliegend, bei den Aktionsschwingungen auf Augenbelichtung daran zu nungen der Hirnrindenfelder, Leipzig Thi e me 1937, S. 36 .

${ }_{28}$ Interessante Oszillogramme hierzu: W. B ü r c k u. H. Li chte, Elektr. Nachr.-Techn. 15, 78 [1938]. Über Frequenzspektren von Spannungsstößen: J. W a ll ot, Einführung in die Theorie der Schwachstromtechnik, Berlin, Springer 1943, S. 383. denken, daß Schaltstöße auf Schleichwegen über den Verstärker auf das Filter gekommen sind. Die Versuchsbedingungen waren aber derart, $\mathrm{da} ß$ die Lichtquelle gar nicht abgeschaltet, sondern nur abgedeckt wurde. Im übrigen kann man aus den erhaltenen Kurven selbst Rückschlüsse auf die erregende Ursache ziehen. Technische Schaltstöße liefern bei Siebung mit einem SelektivFilter in allen Einstellungen gedämpft abklingende Sinusschwingungen (Maximalamplitude beim Einsetzen und Abklingen nach einer Exponentialfunktion). Derartige Kurvenbilder sind bei gefilterten EEG-Aufnahmen überhaupt nicht zur Darstellung gekommen. Bei vielen der höher frequenten Komponenten wurden Schwingungen aufgezeichnet, die nicht der Resonanzfrequenz entsprachen; z. B. $60 \mathrm{~Hz}$ bei Einstellung des Filters auf $56 \mathrm{~Hz}$, $88 \mathrm{~Hz}$ bei Einstellung auf $77 \mathrm{~Hz}$ und $107 \mathrm{~Hz}$ bei Einstellung auf $112 \mathrm{~Hz}$.

Neben der bereits mehrfach erwähnten Ausschaltung der Störkomponenten ergibt sich noch eine weitere praktische Nutzanwendung bei der bisher üblichen ungefilterten Aufnahme des EEG. Diese betraf bisher im wesentlichen das Verhalten der $\alpha$-Welle, $\alpha$-Aktivierung, $\alpha$-Reduzierung, regionäre $\alpha$-Unterschiede, welche gefiltert wesentlich klarer und unverfälschter zutage treten.

Nachdem ein gewisser Überblick über die interessierenden Frequenzen vorhanden ist, werden Filter mit andersartigen Übertragungsbereichen noch eine wichtige Rolle spielen können. Eine Brummsperre, deren sonst lineares Übertragungsband bei $50 \mathrm{~Hz}$ einen Sperrbereich hat, wird die Schwierigkeiten wesentlich verringern, die durch die Einstreuung des Netzbrumm entstehen. Ähnliche Filter zur Unterdrückung der Hauptfrequenzen der Muskelaktionsströme werden ebenfalls erhebliche Erleichterungen bei der EEG-Aufnahme bringen können. Wesentliche Fortschritte für die EEG-Forschung versprechen wir uns, wenn erst allgemein das EEG über einen „Hochpaß“ aufgenommen wird, der nur die Frequenzen oberhalb $10 \mathrm{~Hz}$ überträgt. Je nach der Fragestellung wird man auch mit Tiefpässen ${ }^{30}$ oder Filtern arbeiten, die bei $10 \mathrm{~Hz}$ einen Sperrbereich haben. Wenn so die blendende $\alpha$-Schwingung unterdrückt wird, werden die übrigen Bestandteile des EEG für die Forschung freigelegt werden und uns sicher noch viele wichtige Aufschlüsse liefern.

29 Man betrachte zum Vergleich Oszillogramme des Vokals ,a“ und seiner Komponenten, die durchaus ähnliche Verhältnisse wie beim EEG ergeben. F. Trendelenburg, Klänge und Geräusche, Berlin Spring e r 1935 , S. 84 .

30 B. Frane k u. J. Pras t, Elektrische Narkoseüberwachung, Klinik u. Praxis 1, 112 [1946]. 